\title{
ON BASICITY OF EIGENFUNCTIONS OF SECOND ORDER DISCONTINUOUS DIFFERENTIAL OPERATOR
}

\author{
B.T. BILALOV, T.B. GASYMOV
}

\begin{abstract}
We consider a spectral problem for a second order discontinuous differential operator with spectral parameter in the boundary condition. We present a method for establishing the basicity of eigenfunctions for such problem. We also consider a direct expansion of a Banach space with respect to subspaces and we propose a method for constructing a basis for a space by the bases in subspaces. We also consider the cases when the bases for subspaces are isomorphic and the corresponding isomorphisms are not needed. The completeness, minimality and uniform minimality of the corresponding systems are studied. This approach has extensive applications in the spectral theory of discontinuous differential operators.
\end{abstract}

Keywords: eigenfunctions, basis, completeness, minimality, uniform minimality.

\section{INTRODUCTION}

The study of the spectral properties of many discrete differential operators requires new methods for constructing bases. This was the motivations for many mathematicians to study intensively the basis properties (such as completeness, minimality, basicity) of the systems of special functions, mostly eigenfunctions and associated functions of differential operators. Various methods were developed for establishing these properties. For more information, we refer the reader to [1, 2, 3, 4, 5, 6, 7, 8, 9].

In the case of a discontinuous differential operator, there arise the systems of eigenfunctions whose basicity can not be treated by the standard methods. To shed some light on this situation, we consider the following model spectral problem for a second order discontinuous differential operator

$$
-y^{\prime \prime}(x)=\lambda y(x), x \in(-1,0) \cup(0,1),
$$

with the boundary conditions

$$
y(-1)=y(1)=0, \quad y(-0)=y(+0), \quad y^{\prime}(-0)-y(+0)=\lambda m y(0) .
$$

This spectral problem has two sets of the eigenfunctions [10]:

$$
u_{1 n}(x)=\sin \pi n x, x \in[-1,1], n \in \mathbb{N},
$$

and

$$
\tilde{u}_{2 n}(x)=\left\{\begin{aligned}
\sin \pi n x+o\left(\frac{1}{n}\right), & x \in[-1,0], \\
-\sin \pi n x+o\left(\frac{1}{n}\right), & x \in[0,1], \quad n \in \mathbb{N} .
\end{aligned}\right.
$$

B.T. Bilalov, T.B. Gasymov. On basicity of Eigenfunctions of Second order Discontinuous DIFFERENTIAL OPERATOR.

C.B.T. Bilalov, T.B. Gasymov 2017.

This work was supported by the Research Program Competition launched by the National Academy of Sciences of Azerbaijan ( Program: Frame theory Applications of Wavelet Analysis to Signal Processing in Seismology and Other Fields).

Submitted February 13, 2016. 
Such spectral problems arise while one solves the problem of a loaded string fixed at both ends with a load placed in the middle of the string by the Fourier method [11, 12]. The use of this method requires the study of basis properties of the double system $\left\{u_{1 n} ; \tilde{u}_{2 n}\right\}_{n \in \mathbb{N}}$ in corresponding spaces of functions (usually in the Lebesgue or Sobolev spaces). Of course, it should be started with the basis properties of the system $\left\{u_{1 n} ; u_{2 n}\right\}_{n \in \mathbb{N}}$, which is the principal part of the asymptotics of the system $\left\{u_{1 n} ; \tilde{u}_{2 n}\right\}_{n \in \mathbb{N}}$ :

$$
u_{2 n}(x)=\left\{\begin{aligned}
\sin \pi n x, & {[-1,0), } \\
-\sin \pi n x, & x \in[0,1] .
\end{aligned}\right.
$$

This is usually done by applying various perturbation methods. This approach is well studied (see, e.g., the articles [6, 7, 8, 9, 14, 15, 16, 17, 18, 19, 28, 29, 30, 31] and the monographs [13, 20, 21, 22, 23, 24]). On the other hand, it is not difficult to see that the principal part $\left\{u_{1 n} ; \vartheta_{n}\right\}_{n \in \mathbb{N}}$ is not a standard (in other words, classical) system. It turns out that the form of the system $\left\{u_{1 n} ; \vartheta_{n}\right\}_{n \in \mathbb{N}}$ is not special, i.e. it can be derived from the general case. A general approach applied to these systems allows one to introduce a new method for constructing bases with a lot of applications in the spectral theory of differential operators.

In this work, we consider an abstract approach to the above problem. We consider a direct expansion of a Banach space with respect to subspaces. We propose a method for constructing a basis for a space by means of the bases in the subspaces. We also consider the cases, when the bases in the subspaces are isomorphic and the corresponding isomorphisms may not hold. And we study the completeness, the minimality and the uniform minimality of the corresponding systems. The obtained results are applied to proving the basicity for the eigenfunctions of a second order discontinuous differential operator with the spectral parameter in the boundary condition.

\section{NOTATION AND PRELIMINARIES}

We will use the standard notation. Namely, $\mathbb{N}$ is a set of all positive integers, $L[M]$ denotes the linear span of the set $M$ and $\bar{M}$ stands for the closure of $M ; X^{*}$ denotes the dual space for $X$. Let $L\left(X_{1}, X_{2}\right)$ be a space of linear bounded operators from $X_{1}$ into $X_{2}$ with $L(X, X)=L(X)$, $D_{T}$ be the domain of an operator $T$ and $R_{T}$ be the range of $T$. Let Ker $T$ stand for the kernel of an operator $T,\langle x, f\rangle=f(x)$ denote the value of a functional $f$ at a point $x$. Banach space are referred to as $B$-space; Hilbert space is referred to as $H$-space; $\|\cdot\|_{X}$ denotes a norm in $X ; \Leftrightarrow$ means "if and only if"; $1: n \equiv\{1 ; \ldots ; n\} ; \delta_{i j}$ is the Kronecker delta.

Let us recall the definitions of completeness, minimality, uniform minimality, basicity and basicity with parentheses of a system in a $B$-space.

Let $X$ be some $B$-space. A system $\left\{u_{n}\right\}_{n \in \mathbb{N}} \subset X$ is called complete in $X$ if $\overline{L\left[\left\{u_{n}\right\}_{n \in \mathbb{N}}\right]}=X$.

Using the Hahn-Banach theorem, it is easy to prove the validity of the following completeness criterion.

Completeness criterion. Let $X$ be a normed space. A system $\left\{x_{n}\right\}_{n \in \mathbb{N}} \subset X$ is complete in $X$ if and only if for all $f \in X^{*}:\left\langle x_{n}, f\right\rangle=0$ for each $n \in \mathbb{N}$, implies $f=0$.

A system $\left\{x_{n}\right\}_{n \in \mathbb{N}} \subset X$ is called minimal in $X$ if

$$
x_{k} \notin \overline{L\left[\left\{x_{n}\right\}_{n \in \mathbb{N}_{k}}\right]} \text { for all } k \in \mathbb{N}, \quad \text { where } \quad \mathbb{N}_{k}=\mathbb{N} \backslash\{k\} .
$$

Systems $\left\{x_{n}\right\}_{n \in \mathbb{N}} \subset X$ and $\left\{x_{n}^{*}\right\}_{n \in \mathbb{N}} \subset X^{*}$ are called biorthogonal if $\left\langle x_{m}, x_{n}^{*}\right\rangle=\delta_{n m}$ for all $n, m \in \mathbb{N}$.

The following minimality criterion is available in many monographs.

Minimality criterion. A system in a B-space is minimal if and only if it has a biorthogonal system.

The following basicity criterion in $B$-spaces is true. 
Basicity criterion. A system $\left\{x_{n}\right\}_{n \in \mathbb{N}} \subset X$ forms a basis for a $B$-space $X$ if and only if the following conditions are satisfied:

1) $\left\{x_{n}\right\}_{n \in \mathbb{N}}$ is complete in $X$;

2) $\left\{x_{n}\right\}_{n \in \mathbb{N}}$ is minimal in $X$;

3) The projectors $P_{m}(\cdot)=\sum_{n=1}^{m}\left\langle\cdot, x_{n}^{*}\right\rangle x_{n}$ are uniformly bounded, i.e., there exists $M>0$ such that

$$
\left\|P_{m} x\right\|_{X} \leqslant M\|x\|_{X}, \quad \forall x \in X
$$

where $\left\{x_{n}^{*}\right\}_{n \in \mathbb{N}} \subset X^{*}$ is a system biorthogonal to $\left\{x_{n}\right\}_{n \in \mathbb{N}}$.

A system $\left\{x_{n}\right\}_{n \in \mathbb{N}} \subset X$ is called uniformly minimal in $X$ if

$$
\exists \delta>0: \inf _{\forall u \in L\left[\left\{x_{n}\right\}_{n \neq k}\right]}\left\|x_{k}-u\right\|_{X} \geqslant \delta\left\|x_{k}\right\|_{X}, \forall k \in \mathbb{N} .
$$

Uniform minimality criterion. A complete system $\left\{x_{n}\right\}_{n \in \mathbb{N}} \subset X$ is uniformly minimal in $X$ if and only if $\sup \left\|x_{n}\right\|_{X}\left\|x_{n}^{*}\right\|_{X^{*}}<+\infty$, where $\left\{x_{n}^{*}\right\}_{n \in \mathbb{N}} \subset X^{*}$ is the biorthogonal system.

If a system $\left\{x_{n}\right\}_{n \in \mathbb{N}} \subset X$ forms a basis in $X$, then it is uniformly minimal.

We will also need the concept of basicity with parentheses.

A system $\left\{x_{n}\right\}_{n \in \mathbb{N}} \subset X$ is called a basis with parentheses for $X$ if there exists a sequence of indices $\left\{n_{k}\right\}_{k \in \mathbb{N}} \subset \mathbb{N}: n_{k}<n_{k+1}, \forall k \in \mathbb{N}$, such that every $x \in X$ can be uniquely expanded in a series

$$
x=\sum_{k=0}^{\infty} \sum_{j=n_{k}+1}^{n_{k+1}} c_{j} x_{j}, \quad n_{0}=0 .
$$

Basicity with parentheses criterion. A system $\left\{x_{n}\right\}_{n \in \mathbb{N}}$ forms a basis with parentheses for a $B$-space $X$ if and only if the following conditions are satisfied:

1) $\left\{x_{n}\right\}_{n \in \mathbb{N}}$ is complete in $X$;

2) $\left\{x_{n}\right\}_{n \in \mathbb{N}}$ is minimal in $X$;

3) there exists a sequence of indices $\left\{n_{k}\right\}_{k \in \mathbb{N}} \subset \mathbb{N}$ obeying $n_{k}<n_{k+1}$ for all $k \in \mathbb{N}$ such that the projectors $Q_{k}(\cdot)=\sum_{n=1}^{n_{k}}\left\langle\cdot, x_{n}^{*}\right\rangle x_{n}$ are uniformly bounded, i.e., there exists $M>0$ such that

$$
\left\|Q_{k} x\right\|_{X} \leqslant M\|x\|_{X} \quad \text { for all } \quad x \in X
$$

where $\left\{x_{n}^{*}\right\}_{n \in \mathbb{N}} \subset X^{*}$ is a system biorthogonal to $\left\{x_{n}\right\}_{n \in \mathbb{N}}$.

For more information we refer the reader to the monographs [20, 21, 22, 23, 24, 25, 26, 27].

The following easy-to-prove lemma is often used in the spectral theory of differential operators.

Lemma 1. Let the system $\left\{x_{n}\right\}_{n \in \mathbb{N}}$ form a basis with parentheses for $X$. If the system $\left\{x_{n}\right\}_{n \in \mathbb{N}}$ is uniformly minimal and the sequence $\left\{n_{k+1}-n_{k}\right\}_{k \in \mathbb{N}}$ is bounded, then it forms a usual basis in $X$.

Proof. According to the criterion of basicity with parentheses, the system $\left\{x_{n}\right\}_{n \in \mathbb{N}}$ is complete and minimal in $X$, and the projectors $\left\{P_{n_{k}}\right\}_{k \in \mathbb{N}}$ are uniformly bounded, where $\left\{x_{n}^{*}\right\}_{n \in \mathbb{N}} \subset X$ is the biorthogonal system for $\left\{x_{n}\right\}_{n \in \mathbb{N}}$ )

$$
P_{n_{k}} x=\sum_{n=1}^{n_{k}}\left\langle x, x_{n}^{*}\right\rangle x_{n} .
$$

Therefore it suffices to show that the projectors $\left\{P_{n}\right\}_{n \in \mathbb{N}}$ are uniformly bounded. Let $n_{k}<n<n_{k+1}$. Then

$$
P_{n} x=P_{n_{k}} x+\sum_{i=n_{k}+1}^{n}\left\langle x, x_{i}^{*}\right\rangle x_{i} .
$$


Hence, we have

$$
\begin{aligned}
\left\|P_{n} x\right\| & \leqslant\left\|P_{n_{k}} x\right\|+\left\|\sum_{i=n_{k}+1}^{n}\left\langle x, x_{i}^{*}\right\rangle x_{i}\right\| \leqslant M\|x\|+\left(n-n_{k}\right) \sup _{n_{k} \leqslant i \leqslant n}\left\|x_{i}^{*}\right\|\left\|x_{i}\right\|\|x\| \\
& \leqslant M\|x\|+\left(n_{k+1}-n_{k}\right) \sup _{i \in \mathbb{N}}\left\|x_{i}^{*}\right\|\left\|x_{i}\right\|\|x\| .
\end{aligned}
$$

By the assumption,

$$
\sup _{k \in \mathbb{N}}\left(n_{k+1}-n_{k}\right)<+\infty, \quad \sup _{i \in \mathbb{N}}\left\|x_{i}^{*}\right\|\left\|x_{i}\right\|<+\infty
$$

Therefore,

$$
\left\|P_{n} x\right\| \leqslant \text { const }\|x\|, \quad n \in \mathbb{N} .
$$

We will also need the concept of an almost normed system.

A system $\left\{x_{n}\right\}_{n \in \mathbb{N}} \subset X$ in a $B$-space $X$ is called almost normed if

$$
0<\inf _{n}\left\|x_{n}\right\|_{X} \leqslant \sup _{n}\left\|x_{n}\right\|_{X}<+\infty .
$$

For a uniformly minimal system $\left\{x_{n}\right\}_{n \in \mathbb{N}}$, the almost normed property is equivalent to the following condition:

$$
\sup _{n \in \mathbb{N}}\left\{\left\|x_{n}\right\| ;\left\|x_{n}^{*}\right\|\right\}<+\infty,
$$

where the dual system $\left\{x_{n}^{*}\right\}_{n \in \mathbb{N}}$ is also almost normed.

A system $\left\{\varphi_{n}\right\}_{n \in \mathbb{N}} \subset X$ is called $\omega$-linearly independent in $X$ if

$$
\sum_{n=1}^{\infty} \lambda_{n} \varphi_{n}=0 \quad \text { in } \quad X
$$

implies $\lambda_{n}=0$ for all $n \in \mathbb{N}$.

In the sequel, we will use the following construction and some obvious facts. Let the following direct sum

$$
X=X_{1} \oplus \ldots \oplus X_{m}
$$

hold, where $X_{i}, i=\overline{1, m}$, are some $B$-spaces. For convenience, we represent the elements in the space $X$ as a vector

$$
x \in X \Leftrightarrow x=\left(x_{1}, x_{2}, \ldots, x_{m}\right),
$$

where $x_{k} \in X_{k}, k=\overline{1, m}$. The norm in $X$ is defined by the formula

$$
\|x\|_{X}=\sqrt{\sum_{i=1}^{m}\left\|x_{i}\right\|_{X_{i}}^{2}} .
$$

Then we have $X^{*}=X_{1}^{*} \oplus \ldots \oplus X_{m}^{*}$ (see [13]), and for $f \in X^{*}$ and $x \in X$ the identity

$$
<x, f>=\sum_{i=1}^{m}<x_{i}, f_{i}>
$$

holds true, where $f=\left(f_{1}, \ldots, f_{m}\right)$ and

$$
\|f\|_{X}=\sqrt{\sum_{i=1}^{m}\left\|f_{i}\right\|_{X_{i}^{*}}^{2}} .
$$

From now on, we omit the subscripts in the notation for norms. 
Suppose we are given some system $\left\{u_{i n}\right\}_{n \in \mathbb{N}} \subset X_{i}$ for every $i=\overline{1, m}$. We consider the following system in the space $X$ :

$$
u_{i n}^{0}=(\underbrace{0, \ldots, u_{i n}}_{n}, \ldots, 0), i=\overline{1, m} ; n \in \mathbb{N} .
$$

There is a following obvious relationship between the basis properties of these two systems.

Corollary 1. Each of the following properties, completeness, minimality, uniform minimality or $\omega$-linear independence, basicity of the system $\left\{u_{i n}\right\}_{n \in \mathbb{N}}$ in the space $X_{i}$ for every $i=\overline{1, m}$ is equivalent to the same property of the system $\left\{u_{i n}^{0}\right\}_{i=\overline{1, m} ; n \in \mathbb{N}}$ in the space $X$.

We will also use the concept of the space of coefficients. We define it as follows. Let $\vec{x} \equiv\left\{x_{n}\right\}_{n \in \mathbb{N}} \subset X$ be a non-degenerate system in a $B$-space $X$, i.e., $x_{n} \neq 0$ for all $n \in \mathbb{N}$. We define

$$
\mathscr{K}_{\vec{x}} \equiv\left\{\left\{\lambda_{n}\right\}_{n \in \mathbb{N}}: \text { the series } \sum_{n=1}^{\infty} \lambda_{n} x_{n} \text { converges in } X\right\} \text {. }
$$

We introduce the norm in $\mathscr{K}_{\vec{x}}$ :

$$
\|\vec{\lambda}\|_{\mathscr{K}_{\vec{x}}}=\sup _{m}\left\|\sum_{n=1}^{m} \lambda_{n} x_{n}\right\|, \quad \text { where } \quad \vec{\lambda}=\left\{\lambda_{n}\right\}_{n \in \mathbb{N}} .
$$

The space $\mathscr{K}_{\vec{x}}$ equipped by the usual summation and multiplication by complex number is a Banach space. More information about the above facts can be found in [22, 23, 24, 28].

\section{Completeness And minimality}

Let the following direct sum

$$
X=X_{1} \oplus \ldots \oplus X_{m}
$$

hold true, where $X_{i}, i=\overline{1, m}$, are some $B$-spaces, and let some system $\left\{u_{i n}\right\}_{n \in \mathbb{N}}$ be given in the space $X_{i}$ for every $i=\overline{1, m}$. We consider the following system in the space $X$ :

$$
\omega_{i n}=\left(a_{i 1} u_{1 n} ; \ldots ; a_{i m} u_{m n}\right), i=\overline{1, m} ; n \in \mathbb{N},
$$

where $a_{i j}$ are some numbers. Let

$$
A=\left(a_{i j}\right)_{i, j=1, m}, \quad \Delta=\operatorname{det} A .
$$

The following theorem is true.

Theorem 1. Let the system $\left\{u_{i n}\right\}_{n \in \mathbb{N}}$ be complete (minimal) in the space $X_{i}, i=\overline{1, m}$. If $\Delta \neq 0$, then the system $\left\{\omega_{i n}\right\}_{i=\overline{1, m ; n} \in \mathbb{N}}$ is also complete (minimal) in the space $X$.

Proof. Let the system $\left\{u_{i n}\right\}_{n \in \mathbb{N}}$ be complete in $X_{i}, \quad i=\overline{1, m}$. If for some $\vartheta \in X^{*}$

$$
<\omega_{\text {in }}, \vartheta>=0, \quad i=\overline{1, m}, n=1,2, \ldots,
$$

then it follows from the representation $X^{*}=X_{1}^{*} \dot{\oplus} \ldots \oplus X_{m}^{*}$ and $\vartheta=\left(\vartheta_{1}, \ldots, \vartheta_{m}\right), \vartheta_{i} \in X_{i}^{*}$, $i=\overline{1, m}$, that

$$
\sum_{j=1}^{m} a_{i j}<u_{j n}, \vartheta_{j}>=0, \quad i=\overline{1, m} .
$$

As $\Delta=\operatorname{det}\left(a_{i j}\right) \neq 0$, the homogeneous system of linear equations (4) has only the trivial solution for every $n \in \mathbb{N}$ :

$$
<u_{j n}, \vartheta_{j}>=0, \quad j=\overline{1, m}, \quad n \in \mathbb{N} .
$$


As $\vartheta_{j} \in X_{j}^{*}$, the completeness of the system $\left\{u_{j n}\right\}_{n \in \mathbb{N}}$ in $X_{j}$ implies $\vartheta_{j}=0, j=\overline{1, m}$, i.e. $\vartheta=0$.

Now let $\left\{u_{i n}\right\}_{n \in \mathbb{N}}$ be minimal in $X_{i}$, and $\left\{\vartheta_{i n}\right\}_{n \in \mathbb{N}} \subset X_{i}^{*}$ be the biorthogonal system. We consider the following system in $X^{*}$ :

$$
\nu_{i n}=\left(b_{1 i} \vartheta_{1 n} ; b_{2 i} \vartheta_{2 n} ; \ldots ; b_{m i} \vartheta_{m n}\right), \quad i=\overline{1, m}, \quad n \in \mathbb{N},
$$

where the numbers $b_{j i}$ are the elements of the inverse matrix $A^{-1}$. We have

$$
<\omega_{i n}, \nu_{l k}>=\sum_{j=1}^{m} \sum_{s=1}^{m} a_{i j} b_{s l}<u_{n j}, \vartheta_{k s}>=\sum_{j=1}^{m} a_{i j} b_{j l} \delta_{n k}=\delta_{i l} \delta_{n k}, \quad i, l, n, k \in \mathbb{N} .
$$

The latter relation means that the system $\left\{\nu_{i n}\right\}_{i=\overline{1, m ; n} \in \mathbb{N}}$ is biorthogonal for $\left\{\omega_{i n}\right\}_{i=\overline{1, m ; n} \in \mathbb{N}}$, i.e., the system $\left\{\omega_{i n}\right\}_{i=\overline{1, m ; n} \in \mathbb{N}}$ is minimal.

In case $\Delta=0$ we have the following theorem.

Theorem 2. Let the system $\left\{u_{i n}\right\}_{n \in \mathbb{N}}$ be minimal in $X_{i}$ for every $i=\overline{1, m}$. If $\Delta=0$, then the system $\left\{\omega_{i n}\right\}_{i=\overline{1, m ; n} \in \mathbb{N}}$ defined by (3) is not minimal in $X$.

Proof. Let us show that the system $\left\{\omega_{i n}\right\}_{i=\overline{1, m}}$ is linearly dependent for every $n \in \mathbb{N}$. The identity $\operatorname{det}\left(a_{i j}\right)=0$ yields that that there exist numbers $c_{i}, i=\overline{1, m}$, not all equal to zero, such that

$$
\sum_{i=1}^{m} a_{i j} c_{i}=0, j=\overline{1, m}
$$

Then

$$
\sum_{i=1}^{m} c_{i} \omega_{i n}=\sum_{i=1}^{m} c_{i} \sum_{j=1}^{m} a_{i j} u_{j n}^{0}=\sum_{j=1}^{m}\left(\sum_{i=1}^{m} a_{i j} c_{i}\right) u_{j n}^{0}=0 \quad \text { for all } n \in \mathbb{N} .
$$

Thus, in case $\Delta=0$ the system $\left\{u_{i n}\right\}_{i=1, m ; n \in \mathbb{N}}$ is linearly dependent and not minimal.

The following theorem is also true.

Theorem 3. Let the system $\left\{u_{i n}\right\}_{n \in \mathbb{N}}$ be complete and minimal in $X_{i}$ for every $i=\overline{1, m}$. If $\Delta=0$, then the system $\left\{\omega_{i n}\right\}_{i=\overline{1, m ; n} \in \mathbb{N}}$ is not complete and not minimal in $X$.

Proof. Nonminimality of the system $\left\{\omega_{i n}\right\}_{i=\overline{1, m ; n} \in \mathbb{N}}$ in $X$ is implied by the previous theorem. Let us show that it is not complete in $X$. The identity

$$
\Delta=\operatorname{det}\left(a_{i j}\right)=0
$$

yields that there exist the numbers $c_{j}, j=\overline{1, m}$, not all equal to zero, such that

$$
\sum_{j=1}^{m} a_{i j} c_{j}=0, j=\overline{1, m}
$$

Let

$$
u_{j n}^{0}=(\underbrace{0, \ldots, u_{j n}}_{j}, \ldots, 0) \in X, j=\overline{1, m} .
$$

It is not difficult to see that the system $\left\{u_{j n}^{0}\right\}_{j=1, m} ; n \in \mathbb{N}$ is complete and minimal in $X$, and its biorthogonal system is of the form:

$$
\vartheta_{j n}^{0}=\left(0, \ldots, \vartheta_{j n}, \ldots 0\right), \quad j=\overline{1, m} ; n \in \mathbb{N},
$$


where $\left\{\vartheta_{j n}\right\}_{n \in \mathbb{N}} \subset X_{j}^{*}$ is the system biorthogonal to $\left\{u_{j n}\right\}_{n \in \mathbb{N}}$. We consider the functional

$$
\vartheta=\sum_{s=1}^{m} c_{s} \vartheta_{s 1}^{0}
$$

It is clear that $\vartheta \in X^{*}$ and $\vartheta \neq 0$. Let us show that the functional $\vartheta$ vanishes at the system $\left\{\omega_{i n}\right\}$. Indeed, for $n=1$ we have

$$
<\omega_{i 1}, \vartheta>=\sum_{j=1}^{m} a_{i j}<u_{j 1}^{0}, \vartheta>=\sum_{j=1}^{m} a_{i j} \sum_{k=1}^{m} c_{k}<u_{j 1}^{0}, \vartheta_{s 1}^{0}>=\sum_{j=1}^{m} a_{i j} \sum_{s=1}^{m} c_{s} \delta_{j s}=\sum_{j=1}^{m} a_{i j} c_{j}=0 .
$$

For $n \neq 1$ we have

$$
<\omega_{i n}, \vartheta>=\sum_{j=1}^{m} a_{i j} \sum_{s=1}^{m} c_{s}<u_{j n}^{0}, \vartheta_{s 1}>=0 .
$$

Thus, the system $\left\{\omega_{i n}\right\}_{i=\overline{1, m} ; n \in \mathbb{N}}$ is not complete in $X$.

The minimality condition for the system $\left\{u_{i n}\right\}_{n \in \mathbb{N}}$ in the space $X_{i}$ for every $i=\overline{1, m}$ in Theorem 3 is significant, because the system $\left\{\omega_{i n}\right\}_{i=1, m} ; n \in \mathbb{N}$ can be complete in $X$ only if the system $\left\{u_{i n}\right\}_{n \in \mathbb{N}}$ is complete in $X_{i}, i=\overline{1, m}$, and $\Delta=0$. Let us give an example of such a system. Let $X_{1}=X_{2}=X$, where $X$ is some $H$-space with an orthonormal basis $\left\{e_{n}\right\}_{n \in \mathbb{N}}$. Let $u_{i n}=e_{n}, n=1,2, \ldots$, and let the system $\left\{u_{2 n}\right\}_{n \in \mathbb{N}}$ be defined in $X_{2}$ by the following expressions

$$
u_{2 ; n+i}=e_{i}, \quad n=\frac{(k-1) k}{2}, \quad i=\overline{1, k} ; \quad k \in \mathbb{N} .
$$

We note that every element $e_{n}$ appears in this system infinitely many times. We consider the following system in the space $X=X_{1} \oplus X_{2}$ :

$$
\omega_{1 n}=\left(u_{1 n} ; u_{2 n}\right), \omega_{2 n}=(0 ; 0), n \in \mathbb{N} .
$$

Obviously, the matrix corresponding to this system is

$$
A=\left(\begin{array}{ll}
1 & 0 \\
1 & 0
\end{array}\right)
$$

and $\Delta=\operatorname{det} A=0$. Let us show that the system $\left\{\omega_{1 n}\right\}_{n \in \mathbb{N}}$ is complete in $X$. Let $\vartheta \in X$, $\vartheta=\left(\vartheta_{1} ; \vartheta_{2}\right)$, and assume

$$
<\omega_{1 n}, \vartheta>=\left(e_{n}, \vartheta_{1}\right)+\left(u_{2 n}, \vartheta_{2}\right)=0, \quad \forall n \in \mathbb{N} .
$$

Definition of system $\left\{u_{2 n}\right\}$ combined with the relation (6) implies that for every $n \in \mathbb{N}$ there exists a sequence of indices $\left\{n_{k}\right\}$ such $n_{1}<n_{2}<\ldots<n_{k} \rightarrow \infty, k \rightarrow \infty$ and

$$
\left(e_{n}, \vartheta_{1}\right)+\left(e_{n_{k}}, \vartheta_{2}\right)=0, \quad n \in \mathbb{N} .
$$

As $\left(e_{n_{k}}, \vartheta_{2}\right) \rightarrow 0$ when $k \rightarrow \infty$, it follows that

$$
\left(e_{n}, \vartheta_{1}\right)=0, \quad \forall n \in \mathbb{N} .
$$

Consequently, the completeness of the system $\left\{e_{n}\right\}$ implies $\vartheta_{1}=0$. Then from (6) we obtain

$$
\left(u_{2 n}, \vartheta_{2}\right)=0, \quad n \in \mathbb{N} \text {. }
$$

Now it follows from the completeness of the system $\left\{u_{2 n}\right\}_{n \in \mathbb{N}}$ that $\vartheta_{2}=0$, and, as a result, $\vartheta=0$. And in its turn, this means that the system $\left\{\omega_{1 n}\right\}_{n \in \mathbb{N}}$ is complete in $X$. 


\section{BASICITY}

We suppose that the direct expansion $X=X_{1} \oplus \ldots \oplus X_{m}$ holds true, where $X_{k}, k=\overline{1, m}$ are some $B$-spaces. Let $T_{i j}: X_{i} \rightarrow X_{j}$ be some operators. We consider the system

$$
\sum_{i=1}^{m} a_{i j} T_{i j} x_{i}=y_{j}, \quad j=\overline{1, m},
$$

where $y_{j} \in X_{j}, \quad j=\overline{1, m}$, are the given, and $x_{i} \in X_{i}, \quad i=\overline{1, m}$, are the elements to be determined. We assume that the spaces $X_{k}, k=\overline{1, m}$, are pairwise isomorphic and $T_{i j}$ performs a corresponding isomorphism. Besides, we assume that the following conditions are satisfied: $X_{i}$.

A) $T_{i i}=I_{i}, T_{i j}=T_{j i}^{-1}, T_{j k} T_{i j}=T_{i k}$ for all $i, j=\overline{1, m}$, where $I_{i}$ is the identity operator in

Applying the operator $T_{j 1}=T_{1 j}^{-1}$ to the $\mathrm{j}^{\text {th }}$ equation in the system (7), we obtain the following system

$$
\sum_{i=1}^{m} a_{i j} T_{i 1} x_{i}=T_{j 1} y_{j}, \quad j=\overline{1, m} .
$$

Let $\tilde{x}_{i}=T_{i 1} x_{i}, \quad \tilde{y}_{j}=T_{j 1} y_{j}$. It is clear that $\tilde{x}_{i}, \tilde{y}_{j} \in X_{1}$. As a result, we obtain the following system of linear equations in the space $X_{1}$ :

$$
\sum_{i=1}^{m} a_{i j} \tilde{x}_{i}=\tilde{y}_{j}, j=\overline{1, m} .
$$

If the determinant of this system is non-zero, $\Delta=\operatorname{det}\left(a_{i j}\right) \neq 0$, then it is clear that this system is uniquely solvable with respect to $\tilde{x}_{i}$. Then the system (7) is also uniquely solvable.

Thus, the following lemma is true.

Lemma 2. Let the operators $T_{i j}: X_{i} \rightarrow X_{j}$ perform an isomorphism between $X_{i}$ and $X_{j}$, the conditions $A$ ) be satisfied and $\Delta \neq 0$. Then the system (5) is uniquely solvable for each $y \in X, y=\left(y_{1}, \ldots, y_{m}\right)^{t}$, and moreover, there exists $M>0$ :

$$
\|x\|_{X} \leqslant M\|y\|_{X}
$$

where $x=\left(x_{1}, \ldots, x_{m}\right)$.

Estimate (8) is implied immediately by the following representation for the solution of the system (7):

$$
x_{i}=\sum_{j=1}^{m} b_{i j} T_{j i} y_{j}, \quad i=\overline{1, m},
$$

where $b_{i j}$ are the elements of the inverse matrix $A^{-1}$.

We consider the operator $T: X \rightarrow X$ defined by the matrix $\left(a_{i j} T_{i j}\right)_{i, j=1}^{m}$. Let all the conditions of Lemma 2 be satisfied. It follows from this lemma that $\operatorname{Ker} T=\{0\}, R_{T}=X$, and estimate (8) yields $T \in L(X)$. Then it follows from Banach theorem on the inverse operator that $T$ is an automorphism in $X$. Hence, the following theorem is true.

Theorem 4. Let $T_{i j} \in L\left(X_{i}, X_{j}\right)$ be an isomorphism, the conditions $\left.A\right)$ be satisfied and $\Delta \neq 0$. Then the operator $T: X \rightarrow X$ defined by the matrix $\left(a_{i j} T_{i j}\right)_{i, j=1}^{m}$ is an automorphism in $X=X_{1} \oplus \ldots \oplus X_{m}$.

Suppose that the systems $\left\{u_{i n}\right\}_{n \in \mathbb{N}}$ form bases in the spaces $X_{i}, i=\overline{1, m}$, respectively. Let these bases be isomorphic and $T_{i j} \in L\left(X_{i} ; X_{j}\right)$ perform a corresponding isomorphism

$$
T_{i j} u_{i n}=u_{j n}, \quad n \in \mathbb{N} .
$$


It is clear that the spaces of coefficients $\mathscr{K}_{i}, i=\overline{1, m}$, of these bases coincide. And vice versa, if $\mathscr{K}_{i}, \quad i=\overline{1, m}$, coincide, then there exist isomorphisms $T_{i j} \in L\left(X_{i} ; X_{j}\right)$ such that $T_{i j} u_{i n}=u_{j n}, n \in \mathbb{N}$. In addition, conditions A) are satisfied for the operators $T_{i j}$.

We consider the operator $T: X \rightarrow X$ defined by the matrix $\left(a_{i j} T_{i j}\right)_{i, j=\overline{1, m}}$, where $\Delta=$ $\operatorname{det}\left(a_{i j}\right) \neq 0$. It is clear that the system

$$
u_{i n}^{0}=\left(0, \ldots, u_{i n}, \ldots, 0\right), \quad i=\overline{1, m} ; n \in \mathbb{N},
$$

forms a basis in $X$. Then, obviously, the system

$$
\omega_{i n}=T u_{i n}^{0}=\left(a_{i 1} u_{1 n}, a_{i 2} u_{2 n}, \ldots, a_{i m} u_{m n}\right),
$$

also forms a basis in $X$. Hence, the following theorem is true.

Theorem 5. Let the B-spaces $X_{i}, i=\overline{1, m}$, be pairwise isomorphic and the systems $\left\{u_{i n}\right\}_{n \in \mathbb{N}}$ form isomorphic bases in them, respectively. If $\Delta \neq 0$, then the system $\left\{\omega_{i n}\right\}_{i=\overline{1, m} ; n \in \mathbb{N}}$ forms a basis in $X$ isomorphic to the basis $\left\{u_{i n}^{0}\right\}_{i=\overline{1, m} ; n \in \mathbb{N}}$.

Now we consider the case when the isomorphism of subspaces $X_{i}, i=\overline{1, m}$, is not required. As before, we assume that the expansion $X=X_{1} \oplus \ldots \oplus X_{2}$ holds true. Let $\left\{u_{i n}\right\}_{n \in \mathbb{N}}$ form a basis in the space $X_{i}, i=\overline{1, m}$, and the systems $\left\{u_{i n}^{0}\right\}_{i=\overline{1, m} ; n \in \mathbb{N}},\left\{\omega_{i n}\right\}_{i=\overline{1, m} ; n \in \mathbb{N}},\left\{\vartheta_{i n}^{0}\right\}_{i=\overline{1, m} ; n \in \mathbb{N}}$, $\left\{\nu_{i n}\right\}_{i=\overline{1, m} ; n \in \mathbb{N}}$ be defined as in Sections 2 and 3 and by expressions (3), (5). The following theorem is true.

Theorem 6. If $\Delta=\operatorname{det}\left(a_{i j}\right) \neq 0$, then $\left\{\omega_{i n}\right\}_{i=\overline{1, m} ; n \in \mathbb{N}}$ forms a basis with parentheses in the space $X$. If, in addition, the condition

$$
\sup _{i ; n}\left\{\left\|u_{i n}\right\| ;\left\|\vartheta_{i n}\right\|\right\}<+\infty
$$

is satisfied, then the system $\left\{\omega_{i n}\right\}_{i=1, m} ; n \in \mathbb{N}$ forms a usual basis in $X$.

Proof. We represent the system $\left\{\omega_{i n}\right\}$ in the form

$$
u_{i n}=\sum_{j=1}^{m} a_{i j} u_{j n}^{0}, i=\overline{1, m} ; n \in \mathbb{N} .
$$

As we showed in the previous section, the biorthogonal system is of the form

$$
\nu_{i n}=\sum_{j=1}^{m} b_{j i} \vartheta_{j n}^{0}, i=\overline{1, m} ; n \in \mathbb{N},
$$

where the numbers $b_{j i}$ are the elements of inverse matrix $A^{-1}$. It follows that

$$
\begin{aligned}
\sum_{i=1}^{m}<x, \nu_{i n}>\hat{u}_{i n} & =\sum_{i=1}^{m} \sum_{j=1}^{m} \sum_{l=1}^{m} a_{i j} b_{l i}<x, u_{l n}^{0}>u_{j n}^{0} \\
& =\sum_{j=1}^{m} \sum_{l=1}^{m}\left(\sum_{i=1}^{m} b_{l i} a_{i j}\right)<x, \vartheta_{l n}^{0}>u_{j n}^{0} \\
& =\sum_{j=1}^{m} \sum_{l=1}^{m} \delta_{l j}<x, \vartheta_{l n}^{0}>u_{j n}^{0}=\sum_{j=1}^{m}<x, \vartheta_{j n}^{0}>u_{j n}^{0} .
\end{aligned}
$$

Therefore,

$$
S_{N}(x)=\sum_{n=1}^{N} \sum_{i=1}^{m}<x, \nu_{i n}>\omega_{i n}=\sum_{n=1}^{N} \sum_{i=1}^{m}<x, \vartheta_{i n}^{0}>u_{i n}^{0}
$$




$$
=\sum_{i=1}^{m} \sum_{n=1}^{N}<x, \vartheta_{i n}^{0}>u_{i n}^{0} \rightarrow x, \quad \text { as } \quad N \rightarrow \infty .
$$

Thus, the system $\left\{\omega_{i n}\right\}_{i=\overline{1, m} ; n \in \mathbb{N}}$ forms a basis with parentheses in $X$.

Now we assume that the condition

$$
\sup _{i, n}\left\{\left\|u_{i n}\right\| ;\left\|\vartheta_{i n}\right\|\right\}<+\infty
$$

holds true. Then

$$
\sup _{i, n}\left\{\left\|u_{i n}^{0}\right\| ;\left\|\vartheta_{i n}^{0}\right\|\right\}<+\infty,
$$

and by representations $(9)$ and $(10)$ we obtain

$$
\sup _{i, n}\left\{\left\|\omega_{i n}\right\| ;\left\|\nu_{i n}\right\|\right\}<+\infty
$$

Hence, the system $\left\{\omega_{i n}\right\}$ is uniformly minimal and, by basicity with parenthesis criterion, forms a usual basis in $X$.

Remark 1. Under the assumptions of Theorem 5, the spaces of coefficients of the systems $\left\{\omega_{i n}\right\}$ and $\left\{u_{i n}^{0}\right\}$ are isomorphic, and, using the matrix $A$, it is possible to construct explicitly the mapping that performs this isomorphism. In fact, let $\mathscr{K}_{1}$ and $\mathscr{K}_{2}$ be the spaces of coefficients corresponding to the systems $\left\{u_{i n}^{0}\right\}_{i=\overline{1, m}, n \in \mathbb{N}}$ and $\left\{\omega_{i n}\right\}_{i=\overline{1, m} ; n \in \mathbb{N}}$, and $T_{1}$ and $T_{2}$ be the natural isomorphisms, i.e.,

$$
T_{k}: \mathscr{K}_{k} \leftrightarrow X, \quad k=1,2 .
$$

Let $\vec{c}=\left(c_{i n}\right)_{i=\overline{1, m} ; n \in \mathbb{N}} \in \mathscr{K}_{1}, T_{1} \vec{c}=x$ and $\vec{d}=\left(d_{i n}\right)_{i=\overline{1, m} ; n \in \mathbb{N}} \in \mathscr{K}_{2}$ and $T_{2} \vec{d}=x$, where

$$
x=\sum_{i=1}^{m} \sum_{n=1}^{\infty} c_{i n} u_{i n}^{0}, \quad c_{i n}=<x, \vartheta_{i n}^{0}>=\sum_{j=1}^{m} a_{i j}<x, \nu_{j n}>=\sum_{j=1}^{m} a_{i j} d_{j n} .
$$

On the other hand, we have

$$
x=\sum_{n=1}^{m} \sum_{i=1}^{\infty} d_{i n} \omega_{i n}
$$

with

$$
d_{i n}=<x, \nu_{i n}>=\sum_{j=1}^{m} b_{j i}<x, \vartheta_{i n}^{0}>=\sum_{j=1}^{m} b_{j i} c_{j n},
$$

where $A=\left(a_{i j}\right)$ and $A^{-1}=\left(b_{j i}\right)$. Construct the operator $A: \mathscr{K}_{2} \rightarrow \mathscr{K}_{1}$ using the following infinite matrix

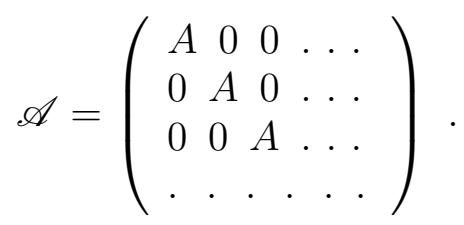

There exists the inverse mapping $\mathscr{A}^{-1}: \mathscr{K}_{2} \rightarrow \mathscr{K}_{1}$ defined by the matrix

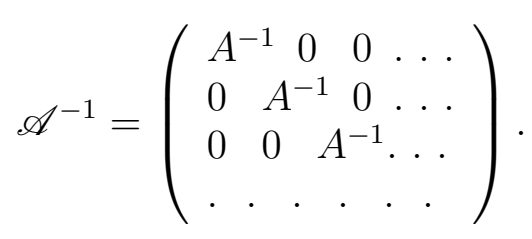


It is clear that for $\vec{c} \in \mathscr{K}_{1}$ we have $\mathscr{A}^{-1} \vec{c}=z$ and for $\vec{d} \in \mathscr{K}_{2}$ we have $\mathscr{A} \vec{d}=y$, i.e., $D_{A}=\mathscr{K}_{2}$, $R_{A}=\mathscr{K}_{1}$. Besides, we have

$$
\begin{aligned}
\left\|T_{1} \vec{c}\right\| & =\|x\|=\left\|\sum_{i=1}^{\infty} \sum_{n=1}^{m}<x, \vartheta_{i n}^{0}>u_{i n}^{0}\right\| \leqslant \sup _{N}\left\|\sum_{n=1}^{N} \sum_{i=1}^{m}<x, \vartheta_{i n}^{0}>u_{i n}^{0}\right\| \\
& \leqslant \sup _{N}\left\|\sum_{n=1}^{N} \sum_{i=1}^{m}<x, \nu_{i n}>\omega_{i n}\right\| \leqslant\|\vec{d}\| .
\end{aligned}
$$

It yields that

$$
\left\|T_{1} A \vec{d}\right\| \leqslant\|\vec{d}\|
$$

i.e. the operator $T_{1} \mathscr{A}=C$ is bounded. The boundedness of the operators $T_{1}, T_{1}^{-1}$ and $C$ implies that $\mathscr{A}$ is bounded. Then, by the Banach theorem on the inverse operator [13, the operator $\mathscr{A}$ is boundedly invertible. Thus, the required isomorphism is constructed.

\section{Application}

Let us apply the obtained general results to the studying the basis properties (completeness, minimality, basicity, Riesz basicity) for the eigenfunctions of problem (1), (2) in $L_{p}(-\pi, \pi)$, $1<p<+\infty$. In order to do it, first we make the following construction.

We identify the spaces $L_{p}(-\pi, 0)$ and $L_{p}(0, \pi)$ with the corresponding subspaces $L_{p}(-\pi, \pi)$, and denote $X_{1} \equiv L_{p}(-\pi, 0), X_{2} \equiv L_{p}(0, \pi)$. We have $X=X_{1} \dot{+} X_{2}$. Thus, each element $f \in L_{p}(-\pi, \pi)$, is identified with the vector $\left(f_{1} ; f_{2}\right)$, where $f_{1}=\left.f\right|_{(-\pi, 0)}, f_{2}=\left.f\right|_{(0, \pi)},\left.f\right|_{M}$ is the restriction of $f$ on $M$. We let $u_{1 n}^{0}=(\sin n x ; 0) ; u_{2 n}^{0}=(0 ; \sin n x), n \in N$. It is clear that the systems $\left\{u_{k n}^{0}\right\}_{n \in N}, k=1,2$, form isomorphic bases in $X_{k}, k=1,2$, respectively. We have

$$
u_{n}^{(1)}=u_{1 n}^{0}+u_{2 n}^{0}, \quad u_{n}^{(2)}=u_{1 n}^{0}-u_{2 n}^{0} \quad \text { for all } n \in N .
$$

Hence, in this case the identities $a_{11}=1, a_{12}=1, a_{21}=1, a_{22}=-1$ are true and as a result, $\operatorname{det}\left(a_{i j}\right)_{i, j=1,2} \neq 0$. Then Theorem 5 implies that the system $\left\{u_{n}^{(1)} ; u_{n}^{(2)}\right\}_{n \in N}$ forms a basis in $L_{p}(-\pi, \pi)$. Thus, the following corollary is true.

Corollary 2. The system $\left\{u_{n}^{(1)} ; u_{n}^{(2)}\right\}_{n \in N}$ forms a basis in $L_{p}(-\pi, \pi), 1<p<+\infty$. Moreover, for the system of eigenfunctions $\left\{u_{n}^{(1)} ; \tilde{u}_{n}^{(2)}\right\}_{n \in N}$ of the problem (1), (2), the following properties are equivalent in $L_{p}(-\pi, \pi)$ : i) complete; ii) minimal; iii) forms a basis; iv) as $p=2$, forms a Riesz basis.

Proof. Let us prove the validity of the second part of the corollary. Namely, let us show that the system $\left\{u_{n}^{(1)} ; u_{n}^{(2)}\right\}_{n \in N}$ satisfies Hausdorff-Young type inequality. We choose $1<p \leqslant 2$, $f \in L_{p}(-\pi, \pi)$ and expand it w.r.t. this basis

$$
f=\sum_{n=1}^{\infty} f_{n}^{(1)} u_{n}^{(1)}+\sum_{n=1}^{\infty} f_{n}^{(2)} u_{n}^{(2)},
$$

where $f_{n}^{(k)}$ are the biorthogonal coefficients of the function $f$ w.r.t. system $\left\{u_{n}^{1} ; u_{n}^{2}\right\}_{n \in N}$. The convergence of the series $\sum_{n=1}^{\infty} f_{n}^{(k)} u_{n}^{(k)}, k=1,2$, in $L_{p}(-\pi, \pi)$ implies immediately that the series

$$
\sum_{n=1}^{\infty} f_{n}^{(k)} \sin n x, k=1,2,
$$


also converges in $L_{p}(0, \pi)$. Therefore, by the classical Hausdorff-Young theorem we obtain that

$$
\left\|\left\{f_{n}^{(k)}\right\}_{n \in N}\right\|_{l_{q}} \leqslant c\left\|\sum_{n} f_{n}^{(k)} \sin n x\right\|_{L_{p}(0, \pi)}
$$

where $c>0$ is a constant independent of $f$. Therefore,

$$
\left\|\left\{f_{n}^{(1)}\right\}\right\|_{l_{q}}+\left\|\left\{f_{n}^{(2)}\right\}\right\|_{l_{q}} \leqslant c\left(\left\|\sum_{n} f_{n}^{(1)} \sin n x\right\|_{L_{p}(0, \pi)}+\left\|\sum_{n} f_{n}^{(2)} \sin n x\right\|_{L_{p}(0, \pi)}\right) .
$$

It is easy to see that

$$
\left\|\sum_{n} f_{n}^{(k)} u_{n}^{(k)}\right\|_{L_{p}(-\pi, \pi)} \sim\left\|\sum_{n} f_{n}^{(k)} \sin n x\right\|_{L_{p}(0, \pi)},
$$

i.e., there exists $\delta>0$ such that

$$
\delta\left\|\sum_{n} f_{n}^{(k)} \sin n x\right\|_{L_{p}(0, \pi)} \leqslant\left\|\sum_{n} f_{n}^{(k)} u_{n}^{(k)}\right\|_{L_{p}(-\pi, \pi)} \leqslant \delta^{-1}\left\|\sum_{n} f_{n}^{(k)} \sin n x\right\|_{L_{p}(0, \pi)} .
$$

We let $Y_{k}=\overline{L\left[\left\{u_{n}^{(k)}\right\}_{n \in N}\right]}, k=1,2$. The definition of the double basis implies immediately that there exists the direct decomposition $X=Y_{1} \dot{+} Y_{2}$. Hence, each $f \in X$ has the unique representation $f=f_{1}+f_{2}, f_{k} \in Y_{k}, k=1,2$. Then, as it is known (see e.g. [32]), there exists $m>0$ such that

$$
\left\|f_{1}\right\|_{L_{p}(-\pi, \pi)}+\left\|f_{2}\right\|_{L_{p}(-\pi, \pi)} \leqslant m\|f\|_{L_{p}(-\pi, \pi)} .
$$

In view of this relation, by 12 we obtain

$$
\begin{gathered}
c\left(\left\|\sum_{n} f_{n}^{(1)} \sin n x\right\|_{L_{p}(0, \pi)}+\left\|\sum_{n} f_{n}^{(2)} \sin n x\right\|_{L_{p}(0, \pi)}\right) \leqslant\|f\|_{L_{p}(-\pi, \pi)} \leqslant \\
\leqslant c^{-1}\left(\left\|\sum_{n} f_{n}^{(1)} \sin n x\right\|_{L_{p}(0, \pi)}+\left\|\sum_{n} f_{n}^{(2)} \sin n x\right\|_{L_{p}(0, \pi)}\right) .
\end{gathered}
$$

As a result, by (11) we the following analogue of the Hausdorff-Young theorem

$$
\left\|\left\{f_{n}^{(1)}\right\}\right\|_{l_{q}}+\left\|\left\{f_{n}^{(2)}\right\}\right\|_{l_{q}} \leqslant c\|f\|_{L_{p}(-\pi, \pi)} .
$$

Thus, we have the following statement.

Let $1<p \leqslant 2$. Then for each $f \in L_{p}(-\pi, \pi)$ the inequality

$$
\left\|\left\{f_{n}^{(1)} ; f_{n}^{(2)}\right\}\right\|_{l_{q}} \leqslant c\|f\|_{L_{p}(-\pi, \pi)}
$$

holds true, where $\left\{f_{n}^{(1)} ; f_{n}^{(2)}\right\}$ are the biorthogonal coefficients of $f$ on the system $\left\{u_{n}^{(1)} ; u_{n}^{(2)}\right\}$.

Now, let us consider the system of the eigenfunctions $\left\{\tilde{u}_{n}^{(1)} ; \tilde{u}_{n}^{(2)}\right\}$ of the problem (1), (2), where $\tilde{u}_{n}^{(1)}=u_{n}^{(1)}, \forall n \in N$. Let $\left\{f_{n}^{(1)} ; f_{n}^{(2)}\right\}$ be an arbitrary finite set. We have

$$
\begin{aligned}
\Delta & =\left\|\sum_{n} f_{n}^{(1)}\left(\tilde{u}_{n}^{(1)}-u_{n}^{(1)}\right)+\sum_{n} f_{n}^{(2)}\left(\tilde{u}_{n}^{(2)}-u_{n}^{(2)}\right)\right\|_{L_{p(=\pi, \pi)}} \\
& \leqslant \sum_{n}\left(\left|f_{n}^{(1)}\right|\left\|\tilde{u}_{n}^{(1)}-u_{n}^{(1)}\right\|_{L_{p}(-\pi, \pi)}+\left|f_{n}^{(2)}\right|\left\|\tilde{u}_{n}^{(2)}-u_{n}^{(2)}\right\|_{L_{p}(-\pi, \pi)}\right) \\
& =\sum_{n}\left|f_{n}^{(2)}\right|\left\|\tilde{u}_{n}^{(2)}-u_{n}^{(2)}\right\|_{L_{p}(-\pi, \pi)} .
\end{aligned}
$$


Let $1<p \leqslant 2$. We have

$$
\Delta \leqslant\left\|\left\{f_{n}^{(2)}\right\}\right\|_{l_{q}}\left(\sum_{n}\left\|\tilde{u}_{n}^{(2)}-u_{n}^{(2)}\right\|_{L_{p(-\pi, \pi)}}^{p}\right)^{\frac{1}{p}} \leqslant A\left(\sum_{n}\left\|\tilde{u}_{n}^{(2)}-u_{n}^{(2)}\right\|_{L_{p(-\pi, \pi)}}^{p}\right)^{\frac{1}{p}}\|f\|_{L_{p(-\pi, \pi)}} .
$$

Consider the case $p>2$. In this case the inequality $1<q<2$ is true and again by applying the Hausdorff-Young inequality we obtain

$$
\Delta \leqslant\left\|\left\{f_{n}^{(2)}\right\}\right\|_{l_{p}} I_{q} \leqslant c I_{q}\|f\|_{L_{q}(-\pi, \pi)} \leqslant c I_{q}\|f\|_{L_{p}(-\pi, \pi)},
$$

where

$$
I_{q}=\left(\sum_{n}\left\|\tilde{u}_{n}^{(2)}-u_{n}^{(2)}\right\|_{L_{p}(-\pi, \pi)}^{q}\right)^{1 / q} .
$$

Further arguing is completely analogous to work [6].

\section{REFERENCES}

1. D.L. Russel. On exponential bases for the Sobolev spaces over an interval // J. Math. Anal. Appl. 87:2, 528-550 (1982).

2. A.M. Sedletskii. Approximate properties of exponential systems in Sobolev spaces// Vestn. Mosk. Univ. Ser. Matem. Mekh. 6, 3-8 (1999). (in Russian).

3. Z.G. Huseynov, A.M. Shykhammedov. On bases of sines and cosines in Sobolev spaces// Appl. Math. Lett. 25:3, 275-278 (2012).

4. B.T. Bilalov, T.B. Gasymov. On basicity of a part of a systems with infinite defect// Trans. NAS Azerb. XXVII:7, 53-59 (2007).

5. T.B. Gasymov. On necessary and sufficient conditions of basicity of some defective systems in Banach space// Trans. NAS Azerb. XXVI:1, 65-70 (2006).

6. B.T. Bilalov. Bases of exponentials, sines, and cosines// Differ. Uravn. 39:5, 619-622 (2003).

7. B.T. Bilalov, T.R. Muradov. Defect bases of Banach spaces// Proc. IMM NASA. XXII:XXX, 23-26 (2005).

8. X. He, H. Volkmer. Riesz bases of solutions of Sturm-Liouville equations // J. Fourier Anal. Appl. 7:3, 297-307 (2001).

9. A.A. Huseynli. On the stability of basisness in $L_{p}(1<p<\infty)$ of cosines and sines// Turk. J. Math. 35:1, 47-54 (2011).

10. T.B. Gasymov, Sh.J. Mammadova. On convergence of spectral expansions for one discontinuous problem with spectral parameter in the boundary condition // Trans. NAS Azerb. XXVI:4, 103-116 (2006).

11. A.N. Tikhonov, A.A. Samarskii. Equations of mathematical physics. Izd. Mosc. univ., Moscow (1999). [Int. Ser. Monog. Pure Appl. Mathe. 39, Pregamon Press, Oxford (1963)]

12. F.V. Atkinson. Discrete and continuous boundary problems. Mir, Moscow (1968). (in Russian).

13. T. Kato. Perturbation theory for linear operators. Springer, Berlin (1976).

14. I.C. Gohberg, A.S. Markus. On stability of bases of Banach and Hilbert spaces// Izv. Akad. Nauk. Mold. SSR. 5, 17-35 (1962).

15. V.D. Milman. Geometric theory of Banach spaces. Part I. The theory of basis and minimal systems // Uspekhi Matem. Nauk. 25:3, 113-174 (1970). [Russ. Math. Surv. 25:3, 111-170 (1970).]

16. N.K. Bari. Biorthogonal systems and bases in Hilbert space// Uchen. Zap. Mosk. Gos. Univ. 148, 69-107 (1951).

17. M.I. Kadets. Exact value of Paley-Wiener constant// Dokl. Akad. Nauk SSSR. 6, 1253-1259 (1964). [Sov. Math. Dokl. 5, 559-561 (1964).]

18. B.T. Bilalov. On the basis property of systems of exponentials, cosines, and sines in $L_{p} / /$ Dokl. Math. 365:1, 7-8 (1999).

19. B.T. Bilalov. On bases for some systems of exponentials, cosines, and sines in $L_{p} / /$ Dokl. Math. 379:2, 7-9 (2001). 
20. O. Christensen. An introduction to frames and Riesz bases. Birkhäser, Boston (2003).

21. O. Christensen. Frames, Riesz bases and discrete Gabor-wavelet expansions // Bull Amer. Math. Soc. 38:3, 273-291 (2001).

22. Ch. Heil. A Basis theory Primer. Birkhäuser, Basel (2011).

23. I. Singer. Bases in Banach spaces. V. 1. Springer, Berlin (1970).

24. I. Singer. Bases in Banach spaces. V. 2. Springer, Berlin (1981).

25. I. Gohberg, M.G. Krein. Introduction to the theory of linear nonselfadjoint operators. Transl. Math. Monog. 18. Amer. Math. Soc. Providence, RI (1969).

26. A.S. Markus. Introduction to the spectral theory of polynomial operator pencils // Transl. of Math. Monog. 71. Amer. Math. Soc., Providence RI (1988).

27. R.M. Young. An introduction to nonharmonic Fourier series. Pure Appl. Math. 93. Academic Press, New York (1980).

28. B.T. Bilalov, Z.V. Mamedova. On the frame properties of some degenerate trigonometric system// Dokl. Acad. Nauk. LXVIII:5, 14-18 (2012).

29. B.T. Bilalov, S.R. Sadigova, Z.V. Mamedova. The space of coefficients in a linear topological space // J. Math. Research. 4:6, 83-88 (2012).

30. B.T. Bilalov, Ch.M. Hashimov, On Decomposition In Banach Spaces // Proc. IMM NAS Azerb. 40:2, 97-106 (2014).

31. S.R. Sadigova, Z.A. Kasumov. On atomic decomposition for Hardy classes with respect to degenerate exponential systems // Proc. IMM NAS Azerb. 40:1, 55-67 (2014).

32. W. Rudin. Functional analysis. McGraw-Hill Book Comp., New York (1973).

Bilal Telman oglu Bilalov,

Institute of Mathematics and Mechanics of NAS of Azerbaijan,

9, B. Vahabzade Str.,

AZ1141, Baku, Azerbaijan

E-mail: b_bilalov@mail.ru

Telman Benser oglu Gasymov,

Institute of Mathematics and Mechanics of NAS of Azerbaijan, 9, B. Vahabzade Str., AZ1141, Baku, Azerbaijan 